Frontiers of Information Technology \& Electronic Engineering www.jzus.zju.edu.cn; engineering.cae.cn; www.springerlink.com ISSN 2095-9184 (print); ISSN 2095-9230 (online)

E-mail: jzus@zju.edu.cn

\title{
Erratum:
}

\section{Erratum to: Latent discriminative representation learning for speaker recognition}

\author{
Duolin $\mathrm{HUANG}^{1}$, Qirong MAO ${ }^{11,2}$, Zhongchen $\mathrm{MA}^{1}$, Zhishen $\mathrm{ZHENG}^{1}$, \\ Sidheswar ROUTRAY ${ }^{1}$, Elias-Nii-Noi OCQUAYE ${ }^{1}$ \\ ${ }^{1}$ School of Computer Science and Communication Engineering, Jiangsu University, Zhenjiang 212013, China \\ ${ }^{2}$ Jiangsu Key Laboratory of Security Technology for Industrial Cyberspace, Zhenjiang 212013, China \\ E-mail: 2211708034@stmail.ujs.edu.cn; mao_qr@ujs.edu.cn; zhongchen_ma@ujs.edu.cn; \\ 1209103822@qq.com; sidheswar69@gmail.com; eocquaye@ujs.edu.cn
}

Published online May 22, 2021

https://doi.org/10.1631/FITEE.19e0690

Erratum to: Front Inform Technol Electron Eng, 2021, 22(5):697-708

https://doi.org/10.1631/FITEE.1900690

Unfortunately the fifth author's name was mis-

spelt. It should be Sidheswar ROUTRAY.

The online version of the original article can be found at https://doi.org/10.1631/FITEE.1900690

\footnotetext{
Corresponding author

(D) ORCID: Duolin HUANG, https://orcid.org/0000-0002-3149-2605; Qirong MAO, https://orcid.org/0000-0002-0616-4431

(C) Zhejiang University Press 2021
} 